Tuberkulose 1951;8(suppl 1):I-II

\title{
Contents, Vol. 8, Supplementum 1, 1951
}

Introduction

Origine des malades

PARTIE MÉDICALE

I. - Repartition des malades

II. - Les tuberculeux pulmonaires

Repartition des malades

Causes des décès

Age de la phtisiogénèse

III. - Le destin des tuberculeux pulmonaires

A. - Etude statistique basée sur Гannée d'entrée des «bacillaires» au sanatorium

Léthalité générale 25

Pronostic en fonction de Page à Tentrée au sanatorium . . 34

Repartition des cas et pronostic en fonction de la gravité des lesions

Repartition des cas

Pronostic

44

4. Pronostic en fonction de la bacilloscopie

Destin des «bacillaires» restés positifs à la sortie du sanatorium $(++)$

Destin des «bacillaires» devenus négatifs (-)

50

R. - Etude statistique basée sur Гapparition des premiers symptômes de la maladie chez «les bacillaires»

Léthalité générale 53

Pronostic en fonction de $\Gamma$ âge lors de $\Gamma$ apparition des premiers symptômes pulmonaires 64

Pronostic en fonction de la gravité des lesions et de Гapparition des premiers symptômes 65

C. — Le destin des tuberculeux pulmonaires «fermés»

69

Léthalité générale 70

Pronostic en fonction de Tunilatéralité ou de la bilatéralité des lesions

Page

D. - Tuberculose pulmonaire et localisations tuberculeuses accessoires

Pendant le séjour au sanatorium 75

Localisations tuberculeuses accessoires apparues après le séjour au sanatorium au sanatorium 79

E. - Le traitement sanatorial et ses résultats

Aperçu general

Collapsothérapie
81

86
81 
Durée de la cure

91

F. - Les rechutes

97

Rechutes pulmonaires après la sortie du sanatorium .... $\quad 97$

Rechutes pulmonaires connues par les réponses au questionnaire

Poussées pulmonaires ayant precede celle qui motiva $\Gamma$ entrée

au sanatorium

100

G. - Capacité de travail - Mariage - Maternité

Capacité de travail

109

Nuptialité et natalité

112

Conclusions

115

Resume

123 